\title{
Acute angulation of the aortic arch predisposes a patient to ascending aortic dilatation and aortic regurgitation late after the arterial switch operation for transposition of the great arteries
}

\author{
Gabriella Agnoletti, $\mathrm{PhD},{ }^{a}$ Phalla Ou, $\mathrm{PhD},{ }^{a}$ David S. Celermajer, $\mathrm{PhD},{ }^{\mathrm{b}}$ Younes Boudjemline, $\mathrm{PhD},{ }^{a}$ Davide Marini, $\mathrm{MD},{ }^{a}$ \\ Damien Bonnet, $\mathrm{PhD},{ }^{\mathrm{a}}$ and Yacine Aggoun, $\mathrm{MD}^{\mathrm{C}}$
}

Objective: We assessed the contribution of acute aortic arch angulation and enhanced systolic pulse wave reflection to dilatation of the ascending aorta and aortic regurgitation late after the arterial switch operation for transposition of the great arteries.

Methods: We performed aortography, radial artery applanation tonometry, and transthoracic echocardiography in 47 children (aged 5-6 years) who underwent the arterial switch operation and in 20 matched healthy controls. The aortic arch angle, ratio of ascending/descending aortic diameter, degree of aortic regurgitation, central pulse pressure, aortic augmentation pressure, and augmentation index were measured.

Results: The aortic arch angle was more acute $(55 \pm 6.5$ degrees vs $68 \pm 5$ degrees, respectively, $P<.001)$ and the ratio of the ascending/descending aorta diameter was significantly greater $(1.98 \pm 0.4$ vs $1.55 \pm 0.06$, respectively, $P<.001)$ in the patients who underwent the arterial switch operation compared with controls. Augmentation pressure and augmentation index were higher in the patients who underwent the arterial switch operation than in controls $(7.5 \pm 4.6$ vs $3.4 \pm 5.8$, respectively $P=.04 ; 21$ \pm 10 vs $8 \pm 13$, respectively, $P=.005$ ). A more acute aortic angle was associated with a higher aortic augmentation index $(r=0.41, P<.01)$, a greater ratio of the ascending to descending aorta $(r=-0.6, P<.001)$, and the degree of aortic regurgitation $(r=0.39, P<.01)$.

Conclusion: Sharper angulation of the aortic arch is associated with early pulse wave reflection, dilatation of the ascending aorta, and aortic regurgitation late after the arterial switch operation for transposition of the great arteries.

From the Department of Pediatric Cardiology, UFR Necker-Enfants Malades, University Rene Descartes Paris V, ${ }^{\mathrm{a}}$ Paris, France; Department of Medicine, University of Sydney, ${ }^{\text {b }}$ Sydney, Australia; and Hôpital des Enfants, ${ }^{\mathrm{c}}$ Genève, EU.

Received for publication April 27, 2007; revisions received Aug 18, 2007; accepted for publication Oct 16, 2007.

Address for reprints: Gabriella Agnoletti, $\mathrm{PhD}$, Service de Cardiologie Pédiatrique, Groupe Hospitalier Necker Enfants Malades, AP-HP, 149, rue de Sèvres, 75743 Paris, France, EU (E-mail: gabriella. agnoletti@nck.ap-hop-paris.fr).

J Thorac Cardiovasc Surg 2008;135:568-72 $0022-5223 / \$ 34.00$

Copyright $\odot 2008$ by The American Association for Thoracic Surgery

doi:10.1016/j.jtcvs.2007.10.020
$\mathrm{T}$ The arterial switch operation (ASO) for transposition of the great arteries (TGA) offers the advantage of anatomic correction and provides good early and intermediate results. ${ }^{1}$ The typical long-term complications of atrial correction, such as arrhythmias and dysfunction of the systemic ventricle, are usually avoided. Nevertheless, late clinical concerns include ascending aortic dilatation ${ }^{2}$ and the progressive development of aortic regurgitation (AR) in some children. ${ }^{3}$ We hypothesized that the posterior translocation of the ascending aorta onto the pulmonary valve might create an abnormally acute angulation of the aortic arch, which might in turn lead to enhanced systolic wave reflection, ascending aortic dilatation, or AR.

\section{Patients and Methods}

In the Department of Pediatric Cardiology, Necker-Enfants-Malades, we systematically perform aortography in children 5 to 6 years after ASO. Between June of 2005 and June of 2006, 60 consecutive children with TGA treated by ASO in the neonatal period underwent aortography. Of these patients, 13 were excluded because of potentially confounding diagnoses: aortic coarctation $(n=6)$, bicuspid pulmonary valve $(n=2)$, any previous surgical procedure 


\section{Abbreviations and Acronyms \\ $\mathrm{AR}=$ aortic regurgitation \\ ASO $=$ arterial switch operation \\ TGA $=$ transposition of the great arteries \\ VSD $=$ ventricular septal defect}

to the ascending aorta $(n=2)$, previous pulmonary banding $(n=2)$, and subvalvular aortic stenosis $(n=1)$. Ventricular septal defect (VSD) closed at the time of ASO $(n=12)$ was not considered an exclusion criterion. All patients with TGA underwent a Lecompte maneuver. The median time interval between the ASO and the later evaluation was 5.2 years (range 5-6 years).

Twenty age and weight-matched children scheduled for percutaneous closure of a small ( $\leq 2 \mathrm{~mm}$ ) patent ductus arteriosus constituted the control group. These were children with small, hemodynamically insignificant patent ductus arteriosus, in whom the indication for coil occlusion was to allow us to discontinue the need for periodic examination and infective endocarditis prophylaxis.

The study complied with the Declaration of Helsinki and was approved by the institutional review committee. The parents of all children gave informed consent.

\section{Clinical Data}

Hospital records and surgical files were reviewed. Particular note was made of the degree of discrepancy in size of the great arteries at the preoperative echocardiography and the time of surgery. Aortic and pulmonary annulus and the diameter of both vessels at the sinotubular junction were measured. Discrepancy was graded as absent (including those in whom the aorta was larger than the pulmonary artery); mild (pulmonary artery 1-2 $\mathrm{mm}$ larger than the aorta); moderate (pulmonary artery 3-4 mm larger than the aorta); or severe (pulmonary artery $>4 \mathrm{~mm}$ larger than the aorta).

\section{Cardiovascular Evaluations}

All children had transthoracic echocardiography and aortography. Applanation tonometry (described below) was obtained in all children. Measurements of aortic arch geometry and vascular analyses were performed by 3 blinded technicians.

Aortography. The ascending aorta was measured at its largest diameter, and the descending aorta was measured at the level of diaphragm. The ratio of the ascending to the descending aorta diameter was then obtained. We also quantified the angulation between the ascending and the descending aorta by measuring the aortic arch angle on the lateral aortogram (Figure 1). The midpoints of the ascending (A) and descending (D) aortic diameters were determined at the level of the pulmonary bifurcation, and the highest point $(\mathrm{H})$ of the transverse aorta was identified. The aortic arch angle was measured at the intersection between HA and HD, as shown in Figure 1.

Echocardiography. All children underwent 2-dimensional echocardiography, Doppler investigation, and color Doppler mapping. AR was evaluated by color Doppler and graded as none, trivial, mild, moderate, or severe, as previously described and validated in children. ${ }^{4}$

Applanation tonometry. All children underwent applanation tonometry before aortography. No sedation was administered. The

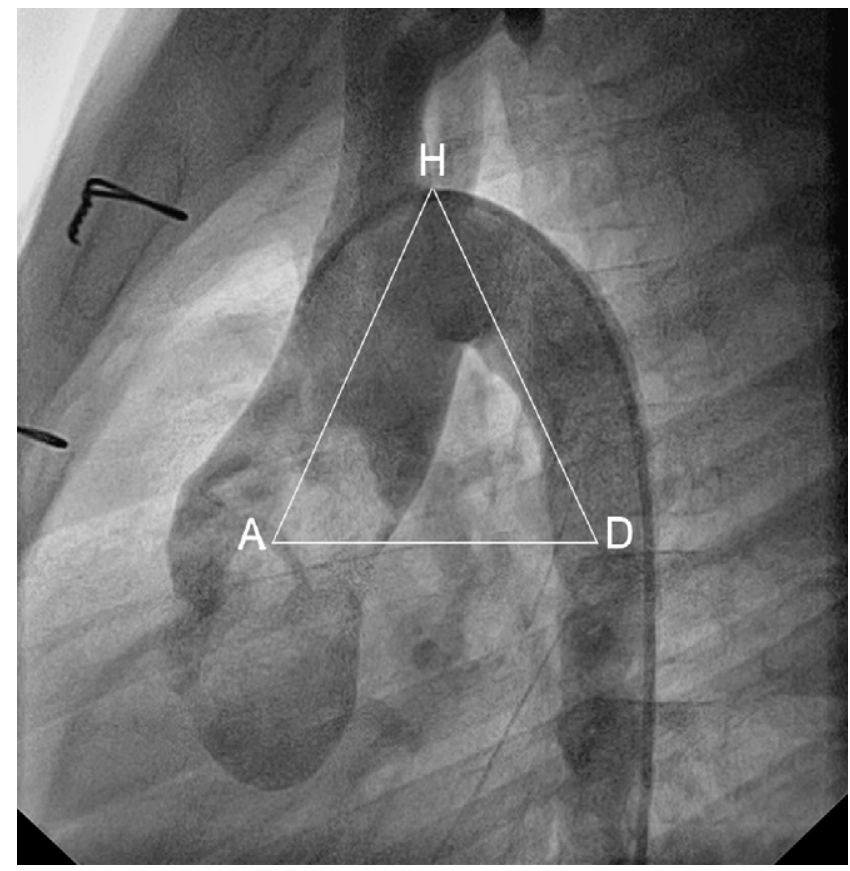

Figure 1. Measurement of the aortic arch angle.

aortic pressure waveform was derived by using a micromanometertipped probe (SphygmoCor; Pulse Wave Medical Ltd, Atcor Medical Inc., Chicago, Ill) applied to the surface of the skin over the right radial artery. The peripheral radial pulse wave was recorded continuously. The SphygmoCor system integrates the radial pulse and the transfer function between the aorta and the radial artery and provides a noninvasive estimate of central aortic pressure. The first systolic inflection (P1), caused by the left ventricular ejection, and the second peak inflection (P2), caused by wave reflection (Figure 2), were extracted from the central aortic pulse and pulse pressure (systolic-diastolic blood pressure) was measured. The difference between the first systolic inflection and the second peak inflection is the degree to which central arterial pressure is augmented by peripheral wave reflection. This increase in pressure is described as the augmentation pressure. The augmentation index corresponds to the augmentation pressure/pulse pressure $\times 100$ (Figure 2). All measurements were calculated as the average of 3 consecutive cardiac cycles. Arterial stiffness was evaluated by using pulse wave velocity, as previously described, by recording pulse pressure at the carotid and femoral sites. ${ }^{5}$

\section{Statistical Analysis}

Descriptive statistics for the total population were obtained. Age and weight were quoted as median and range; continuous variables were presented as the mean \pm standard deviation. Comparison for individual parameters was performed using the 2-tailed unpaired $t$ test. Linear regression analysis was done by using the correlation coefficient $r$. Differences between proportions were evaluated with the chi-square or Fisher exact test.

Independent risk factors for aortic incompetence and dilation of ascending aorta were sought using univariate regression analysis for 


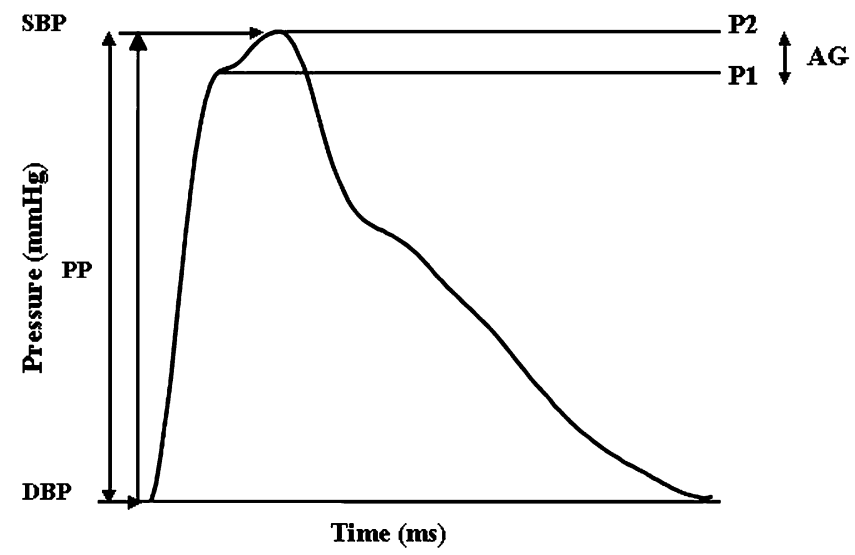

Figure 2. Applanation tonometry. Measurement of pulse pressure and augmentation pressure. $S B P$, Systolic blood pressure; DBP, diastolic blood pressure; $P 1$, first wave inflection; $P 2$, second wave inflection; $A G$, augmentation pressure; $P P$, pulse pressure.

continuous or discrete variables. Statistical analyses were conducted with R software, version 2.00 (Insight Corporation, Boonton, NJ).

\section{Results}

There were 20 girls and 27 boys in the ASO group and 7 girls and 13 boys in the control group. The 2 groups were comparable in terms of age (median value 5 and 5 years, respectively), height (median value 110 and $111 \mathrm{~cm}$, respectively), weight (median value 19 and $19 \mathrm{~kg}$, respectively), and heart rate (median value 84 and 86 beats/min, respectively) (Table 1 ).

\section{Clinic Characteristics and Prevalence of Aortic Regurgitation}

Thirty-five patients had simple TGA and 12 patients had associated VSD. Trivial regurgitation of the native pulmo-

TABLE 1. Clinical parameters, results of vascular analysis, and angiographic parameters

\begin{tabular}{lccc}
\hline \multicolumn{1}{c}{ Parameters } & $\begin{array}{c}\text { TGA (SD) } \\
\mathbf{n}=\mathbf{4 7}\end{array}$ & $\begin{array}{c}\text { Control (SD) } \\
\mathbf{n}=\mathbf{2 0}\end{array}$ & $\begin{array}{c}\boldsymbol{P} \\
\text { value }\end{array}$ \\
\hline Age, y & $5.7 \pm 1.4$ & $5.4 \pm 2.3$ & .60 \\
Height, cm & $111 \pm 12$ & $113 \pm 19$ & .73 \\
Weight, kg & $19 \pm 4$ & $20 \pm 8$ & .55 \\
Central PP, mm Hg & $37 \pm 14$ & $38 \pm 13$ & .86 \\
P1, mm Hg & $30 \pm 12$ & $34 \pm 11$ & .40 \\
AG, mm Hg & $7.4 \pm 4.7$ & $3.4 \pm 5.8$ & .04 \\
Alx & $20 \pm 10$ & $8.3 \pm 13$ & .005 \\
Aortic angle & $55 \pm 6.5$ & $68 \pm 5$ & $<.001$ \\
& degrees & degrees & \\
Ascending/descending & $1.98 \pm 0.4$ & $1.55 \pm 0.06$ & $<.001$ \\
\multicolumn{1}{c}{ aorta diameter } & & & \\
\hline
\end{tabular}

$\overline{T G A}$, Transposition of the great arteries; $S D$, standard deviation; $P P$, pulse pressure; $P 1$, first wave inflection; $A G$, augmentation pressure; $A l x$, augmentation index. nary valve had been noted preoperatively in only 1 neonate. In the immediate postoperative period, mild AR was detected in 8 of the 47 patients. At follow-up, AR was found in 23 patients who underwent the ASO (46\%), including 16 children who had simple TGA (43\%) and 7 of those with associated VSD (58\%). The degree of AR was mild in 17 patients $(74 \%)$, moderate to severe in 6 patients $(26 \%)$, and similar in patients with TGA with and without VSD.

All patients had central AR; 12 patients had a dilated aortic annulus for baby size, ${ }^{6} 3$ of whom had a VSD. The presence and degree of AR were not related to the degree of initial discrepancy between the pulmonary artery and the aortic diameter notes at the time of ASO; indeed, AR was present in 8 of 17 patients without discrepancy, in 11 of 20 patients with mild discrepancy, in 2 of 7 patients with moderate discrepancy, and in 2 of 3 patients with severe arterial size discrepancy. We did not find any correlation between the presence of VSD, analyzed as an independent variable, and the size of the pulmonary artery, possibly because of the limited number of patients with VSD included in the study.

\section{Aortic Arch Geometry}

The degree of angulation of the aortic arch was significantly greater in patients who underwent the ASO than in controls; the aortic arch angle was more acute after the ASO (55 \pm 6.5 degrees vs $68 \pm 5$ degrees, $P<.001$ ) (Figure 3 ). The ratio of the ascending/descending aorta diameter was significantly higher in children who underwent the ASO compared with the controls $(1.98 \pm 0.4$ vs $1.55 \pm 0.06, P<.001)$. The aortic arch angle was negatively correlated with the ratio of the ascending/descending aorta $(r=-0,6, P<.001)$. The ratio of the ascending/descending aorta was significantly correlated with the presence and degree of AR $(r=0.52, r<0.001)$. The degree of initial discrepancy between the pulmonary artery and aortic diameters was not correlated with the ratio of the ascending/descending aorta or with the aortic angle.

\section{Applanation Tonometry}

The heart rate was similar in ASO and control groups (86 \pm 13 beats/min and $86 \pm 11$ beats/min, respectively). Central pulse pressure $(38 \pm 13 \mathrm{~mm} \mathrm{Hg}$ and $37 \pm 15 \mathrm{~mm} \mathrm{Hg}$, respectively) and P1 (31 $\pm 12 \mathrm{~mm} \mathrm{Hg}$ and $34 \pm 12 \mathrm{~mm} \mathrm{Hg}$, respectively) were also similar in the 2 groups. Augmentation pressure was increased in the ASO group compared with controls $(7.5 \pm 4.6 \mathrm{~mm} \mathrm{Hg}$ and $3.4 \pm 5.8 \mathrm{~mm} \mathrm{Hg}$, respectively, $P=.04)$, as well as the augmentation index $(21 \% \pm$ $10 \%$ and $8.3 \% \pm 13 \%$, respectively $P=.005)$. Pulse wave velocity was similar in the 2 groups $(4.6 \pm 0.7 \mathrm{~m} / \mathrm{sec}$ and $4.5 \pm 0.8 \mathrm{~m} / \mathrm{sec}$, respectively).

There was a significant correlation between the aortic angle and augmentation index $(r=0.41, P<.01)$. Augmentation pressure and augmentation index were also significantly 

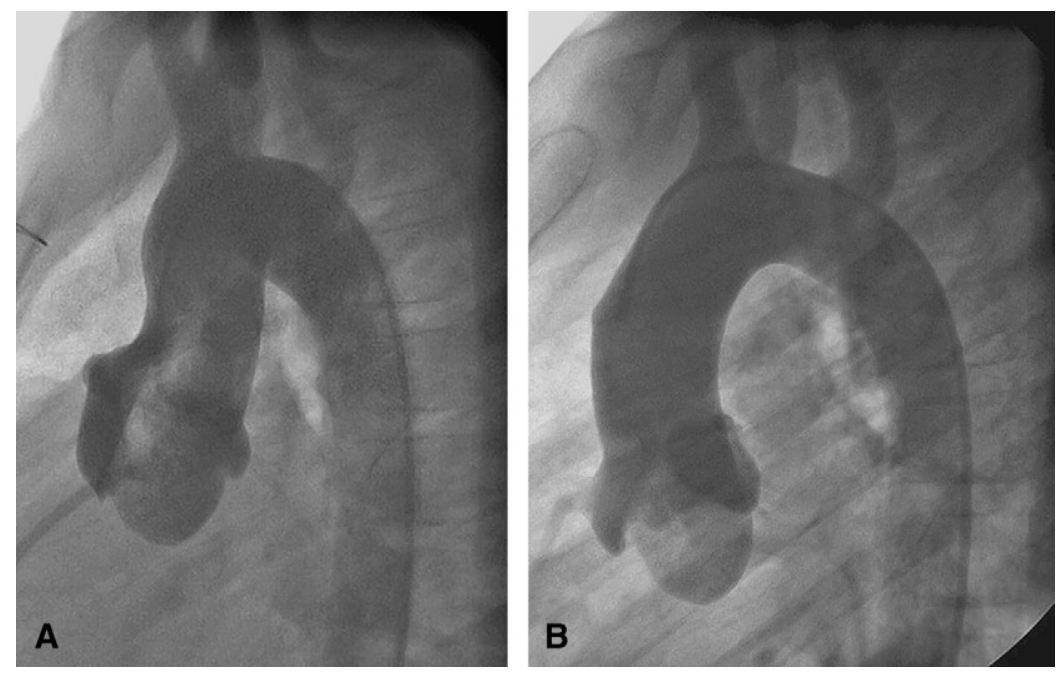

Figure 3. Two examples of aortic arch in a child with ASO (A) and a control child (B).

correlated with the degree of AR $(r=0.56, P<.001$; $r=0.39, P<.01$, respectively). Pulse wave velocity was not correlated with the aortic arch angle.

\section{Discussion}

This study demonstrates that the procedure of posterior translocation of the ascending aorta during the ASO for TGA is associated with acute angulation of the aortic arch, enhanced reflection of the systolic pulse wave as evidenced by an increased augmentation pressure and augmentation index in the ascending aorta, and dilatation of the ascending aorta and AR.

The ASO is currently considered the best surgical option to correct simple TGA. Ascending aortic dilatation and the appearance and progression of $\mathrm{AR}$, however, seem to be potentially serious late complications of this operation., ${ }^{2,7}$ Several factors have been suggested as important in the development of aortic root dilatation, such as previous pulmonary artery banding, VSD, and older age intervention. ${ }^{2,7,8}$ In the current study, we did not find that VSD or great vessel size discrepancy was associated with a higher prevalence of $\mathrm{AR}$, possibly because of the limited number of patients with VSD included in the study; however, we did find a prevalence of AR of $48 \%$ with severe valve regurgitation in approximately $10 \%$ of our subject group.

Our 2 major findings from the applanation tonometry were the increased augmentation pressure and augmentation index found in the children who underwent the ASO. Pulse pressure was similar in the ASO and control groups; thus the presence of AR did not invalidate our results. ${ }^{9}$ In the current setting, we speculate that the more acute aortic angle increases the augmentation indices by providing a site of early wave reflection of the incident systolic wave from the ascending aorta; however, we did not prove this directly. Indeed, we cannot state that the increased angulation of aortic arch is the major factor prompting development of ascending aorta dilatation and aortic incompetence. This would be an informative area for further study, which can now be performed in vivo by advanced imaging techniques, such as magnetic resonance imaging (although this latter would require anesthesia in small children). The progressive dilatation of the ascending aorta and the development of AR in patients who underwent the ASO are probably the results of several factors, not least of which is the section of aortic fibers, which could prompt the loss of aortic wall elasticity. Comparison with patients who underwent a Ross operation could provide further data on the role of aortic surgery in pulse wave propagation, dilatation of ascending aorta, and development of AR.

In our study, we did not find evidence of enhanced arterial stiffness of the ascending aorta, which is often observed in subjects with increased aortic augmentation indices. ${ }^{10} \mathrm{We}$ speculate that this might be due to the young age of these children at the time of the study. Mersich and colleagues ${ }^{11}$ recently documented increased carotid arterial stiffness in patients who underwent the ASO an average of 12 years after surgery.

The limitations of this study include the lack of serial data; thus, we can only present associations among the aortic arch angle, augmentation indices, ascending aortic dilatation, and $\mathrm{AR}$, rather than imputing cause and effect. Furthermore, we were unable to calculate aortic compliance and distensibility measures, in large part because of the young age of the patients. We were unable to find any relation between the presence of VSD or size discrepancy of vessels and the development of AR, probably because of the relatively small number of patients included in the study.

\section{Conclusions}

We found a cluster of aortic abnormalities in healthy children who underwent the ASO for TGA. These include acute angulation of the aortic arch, enhanced pressure wave reflection in 
the ascending aorta, ascending aortic dilatation, and AR. These abnormalities may be consequences of the necessary posterior translocation of the ascending aorta, which is required as part of the ASO but may have deleterious long-term consequences. Animal studies could identify new surgical strategies to limit the deleterious long-term effects of ASO.

\section{References}

1. Hovels-Gurich HH, Seghaye MC, Ma Q, Miskova M, Minkenberg R, Messmer BJ, et al. Long-term results of cardiac and general health status in children after neonatal arterial switch operation. Ann Thorac Surg. 2003;75:935-43.

2. Hourihan M, Colan SD, Wernovsky G, Maheswari U, Mayer JE Jr, Sanders SP. Growth of the aortic anastomosis, annulus, and root after the arterial switch procedure performed in infancy. Circulation. 1993; 88:615-20.

3. Losay J, Touchot A, Capderou A, Piot JD, Belli E, Planche C, et al. Aortic valve regurgitation after arterial switch operation for transposition of the great arteries: incidence, risk factors, and outcome. J Am Coll Cardiol. 2006;47:2057-62.

4. Tani LY, Minich LL, Day RW, Orsmond GS, Shaddy RE. Doppler evaluation of aortic regurgitation in children. Am J Cardiol. 1997;80:927-31.
5. Zureik M, Czernichow S, Courbon D, Blacher J, Ducimetiere P, Hercberg S, et al. Parental longevity, carotid atherosclerosis, and aortic arterial stiffness in adult offspring. Stroke. 2006;37:2702-7.

6. Schwartz ML, Gauvreau K, del Nido P, Mayer JE, Colan SD. Long-term predictors of aortic root dilation and aortic regurgitation after arterial switch operation. Circulation. 2004;110:II128-32.

7. Hwang HY, Kim WH, Kwak JG, Lee JR, Kim YJ, Rho JR, et al Mid-term follow-up of neoaortic regurgitation after the arterial switch operation for transposition of the great arteries. Eur J Cardiothorac Surg. 2006;29:162-7.

8. Hutter PA, Thomeer BJ, Jansen P, Hitchcock JF, Faber JA Meijboom EJ, et al. Fate of the aortic root after arterial switch operation. Eur J Cardiothorac Surg. 2001;20:82-8.

9. Kervancioglu S, Davutoglu V, Ozkur A, Soydinc S, Adaletli I, Sirikci A, et al. Duplex sonography of the carotid arteries in patients with pure aortic regurgitation: pulse waveform and hemodynamic changes and a new indicator of the severity of aortic regurgitation. Acta Radiol. 2004;45 411-6.

10. Agnoletti G, Bonnet C, Bonnet D, Sidi D, Aggoun Y. Mid-term effects of implanting stents for relief of aortic recoarctation on systemic hypertension, carotid mechanical properties, intimal medial thickness and reflection of the pulse wave. Cardiol Young. 2005;15:245-50.

11. Mersich B, Studinger P, Lenard S, Kader K, Kollai. Transposition of great arteries is associated with increased carotid artery stiffness. Hypertension. 2006;47:1197-202. 